\title{
Serum tRNA-derived fragments as potential biomarkers in children with acute intussusception
}

Lian Zhao, Wei Chen, Wan-Liang Guo

Children's Hospital of Soochow University, China

Submitted: 20 April 2021; Accepted: 24 July 2021

Online publication: 27 July 2021

Arch Med Sci

DOI: https://doi.org/10.5114/aoms/140491

Copyright @ 2022 Termedia \& Banach

\section{Abstract}

Introduction: Pediatric intussusception is one of the most common causes of bowel obstruction in the pediatric population. Affected children have one section of the intestine sliding into the adjacent section. Intestinal ischemia-reperfusion injury (I/R) can occur during pediatric intussusception, and any delay in diagnosis or treatment can lead to loss of intestinal viability that requires bowel resection. The aim of the present study was to investigate whether transfer ribonucleic acid (tRNA)-derived fragments (tRFs) can serve as candidate biomarkers for pediatric intussusception.

Material and methods: Using high-throughput sequencing technology, we identified differentially expressed tRFs, and ultimately selected three tRFs to establish a signature as a predictive biomarker of pediatric intussusception. Selection of these three upregulated genes was verified using quantitative reverse-transcription polymerase chain reaction (qRT-PCR). We conducted receiver operator characteristic (ROC) curve analysis to evaluate the predictive accuracy of the selected genes for pediatric intussusception.

Results: We detected 732 tRFs and tRNA-derived stress-induced RNA (tiRNAs), 1705 microRNAs (miRNAs), 52 differentially expressed miRNAs, and 34 differentially expressed tRFs and tiRNAs between patients and controls. Compared with controls, we found 33 upregulated miRNAs, 24 upregulated tRFs and tiRNAs, 19 downregulated miRNAs, and 10 downregulated tRFs and tiRNAs in children with intussusception. Using $\mathrm{qPCR}$, the expression trends of tRF-Leu-TAA-006, tRF-Gln-TTG-033 and tRF-Lys-TTT-028 were consistent with the sequencing results. AUCs of tRF-Leu-TAA-006, tRF-GIn-TTG-033 and tRF-Lys-TTT-028 were $0.984,0.970$ and 0.837 , respectively.

Conclusion: Circulating tRF-Leu-TAA-006, tRF-Gln-TTG-033 and tRF-LysTTT-028 expression might be a novel potential biomarker for diagnosis of pediatric intussusception.

Key words: pediatric, intussusception, intestinal ischemia-reperfusion, gene expression, transfer ribonucleic acid (tRNA)-derived fragments.

\section{Introduction}

Pediatric intussusception is one of the most common causes of bowel obstruction in the pediatric population. Affected children have one section of the intestine sliding into the adjacent section $[1,2]$. Intestinal ischemia-reperfusion injury (I/R) can occur during pediatric intussusception $[3,4]$, and any delay in diagnosis or treatment can lead to loss of intestinal viability that requires bowel resection $[5,6]$.

\author{
Corresponding author: \\ Wan-Liang Guo \\ Children's Hospital \\ of Soochow University \\ 303\#, Jingde Road, \\ 215003, Suzhou, China \\ Phone: +8615950011521 \\ E-mail: gwlsuzhou@163.com
}


In clinical practice, the diagnosis of pediatric intussusception is mainly based on a combination of clinical symptoms and examinations from ultrasound (US) imaging, computed tomography (CT), or air enema [7]. Early detection of pediatric intussusception and associated intestinal I/R can improve prognosis and treatment efficacy $[8,9]$. Until now, there has been no clinical biomarker for early diagnosis of pediatric intussusception and associated intestinal I/R.

Transfer ribonucleic acid (tRNA)-derived fragments (tRFs), a novel type of small non-coding RNA originating from tRNAs $[10,11]$, participate in many pathological processes [12]. These fragments can be released and detected in the peripheral circulation. Levels of tRFs can fluctuate in response to stimuli. Circulating levels of tRFs might be useful as surrogate measurements of disease progression [13, 14]. Mishima et al. reported that in pathological stress damage, such as ischemic reperfusion, toxic injury, and irradiation, the levels of circulating tRNA derivatives showed a rapid increase [15]. However, no previous study has investigated the role of tRFs and tRNA-derived stress-induced RNA (tiRNAs) in pediatric intussusception and associated intestinal I/R. In our previous studies, we found I/R in both our animal models and our pediatric intussusception patients. We also found that $I / R$ is associated with upregulation of p38MAPK in intussusception $[3,4,6]$. In the current study, we examined whether a gene expression profile could be used for early detection of pediatric intussusception. Specifically, we conducted genome-wide transcriptional profiling using blood-derived tRFs and tiRNAs followed by real-time polymerase chain reaction $(P C R)$ verification in children with intussusception.

\section{Material and methods}

\section{Material}

We performed a cross-sectional study. The study protocol was approved by the Ethics Committee of our hospital and complied with Helsinki Declaration. Written informed consent was obtained from their parents. Demographic and clinical data were recorded for each study participant between $\mathrm{Au}$ gust 1, 2019 and December 31, 2019 at our hospital. The diagnosis of intussusception was based on combined clinical symptom with the US examination. Once the diagnosis was made, air enema was performed as the treatment method.

\section{RNA extraction}

Peripheral blood samples were drawn immediately after the final diagnosis by air enema before reduction of intussusception and mononuclear cells were isolated and then stored at $-80^{\circ} \mathrm{C}$ for
RNA extraction. We isolated total RNA using TRIzol Reagent (Invitrogen, Carlsbad, California, US) per the manufacturer's protocol.

\section{Library preparation and tRF/tiRNA sequencing and data analysis}

We used agarose electrophoresis to check the integrality of total RNA samples, and then quantified the samples using a NanoDrop ND-1000 Spectrophotometer (Thermo Scientific, Thermo Fisher Scientific, Waltham, Massachusetts, USA). In order to remove RNA modifications that interfere with small-RNA sequencing (RNA-seq) library construction, total RNA samples were first pretreated as follows: 3-aminoacyl (charged) deacylation to 3-OH for 3-adaptor ligation; 3-cP (2,3-cyclic phosphate) removal to $3-\mathrm{OH}$ for 3-adaptor ligation; 5-OH (hydroxyl group) phosphorylation to 5-P for 5-adaptor ligation; and $\mathrm{m} 1 \mathrm{~A}$ and $\mathrm{m} 3 \mathrm{C}$ demethylation for efficient reverse transcription. We took pretreated total RNA from each sample for tRF and tiRNA sequencing (tRF-seq, tiRNA-seq) library preparation. The procedures included (1) 3-adapter ligation; (2) 5-adapter ligation; (3) complementary deoxyribonucleic acid (cDNA) synthesis; (4) PCR amplification; and (5) size selection of 134-160-bp PCR amplified fragments (corresponding to $\sim 14-40$-nt small RNAs). The completed libraries were quantified using an Agilent 2100 Bioanalyzer (Agilent Technologies, Inc., Santa Clara, California, US). We mixed the libraries in equal amounts according to the quantification results and used them for further sequencing.

We denatured DNA fragments in well-mixed libraries with $0.1 \mathrm{M} \mathrm{NaOH}$ to generate single-stranded DNA molecules and then loaded them onto the reagent cartridge at a concentration of $1.8 \mathrm{pM}$. The sequencing was performed on a NextSeq system using a NextSeq 500/550 V2 kit (\#FC-404-2005; Illumina, Inc., San Diego, California, USA) per the manufacturer's instructions. We performed 50 cycles of sequencing.

We analyzed images and performed base calling using Solexa Pipeline v1.8 software (Off-Line Basecaller software v1.8; Illumina). Sequencing quality was examined using FastQC software [16]. We aligned trimmed reads (pass Illumina quality filter, trimmed 5,3-adaptor bases by cut adapt [17]), allowing for only one mismatch to mature tRNA sequences. Then, we aligned the reads that did not map using Bowtie software [18], allowing for only one mismatch to precursor tRNA sequences. The remaining reads were aligned using miRDeep2 software [19], allowing for only one mismatch to microRNA (miRNA) reference sequences. We were then able to calculate the expression profiling of tRFs, tiRNA, and miRNA based on counts of reads mapped. Differentially expressed 
tRFs, tiRNAs and miRNAs were screened based on the count value using the edgeR package in $\mathrm{R}$ software [20]. We performed principal component analysis (PCA), correlation analysis, and hierarchical clustering to create pie, Venn, scatter, and volcano plots in an R or Perl environment for statistical computing and graphics of the expressed tRFs and tiRNAs. The functions and pathways of almost all tRFs were classified according to Gene Ontology (GO) assignment and the Kyoto Encyclopedia of Genes and Genomes (KEGG) database. We used Cytoscape software (version 3.8.0, https://cytoscape.org/) to predict the network of the top 100 potential targets of tRFs and tiRNAs.

Real-time quantitative reverse transcription polymerase chain reaction assay

Using quantitative reverse-transcription polymerase chain reaction ( $q R T-P C R$ ), we selected and quantified the upregulated expression of tRF-LeuTAA-006, tRF-Gln-TTG-033, and tRF-Lys-TTT-028 in both study groups. We performed GPCR using a SYBR Green PCR Kit (Applied Biosystems, Foster City, California, USA) per the manufacturer's instructions. We normalized gene expression to $\beta$-actin messenger RNA (mRNA). Relative expression of the gene transcript was calculated using the $2^{-\Delta \Delta \mathrm{ct}}$ method.

\section{Statistical analysis}

We used SPSS software (version 20.0, IBM, Armonk, New York, USA) for statistical analysis. Continuous data are presented as mean \pm standard deviation (SD). Inter-group comparisons were performed by the rank sum test. Receiver operator characteristic (ROC) curve analysis was conducted and the area under the ROC curve (AUC) was calculated to evaluate the diagnostic accuracy of selected genes in pediatric intussusception. $P<0.05$ was considered statistically significant.

\section{Results}

\section{Demographic and clinical data}

A total of 20 pediatric-intussusception patients and 20 healthy controls were included in the study. Patients' demographic and clinical data are summarized in Table I. There was no statistically significant difference between the children with intussusception and healthy controls in terms of age (months, $30.97 \pm 34.94$ vs. $35.21 \pm 40.78$, $p=0.511$ ) and gender (male, 10 vs. $12, p=0.781$ ).

\section{Differentially expressed serum tRF and tiRNA levels in patients and controls}

In this study, we detected 732 tRFs and tiRNAs, 1705 miRNAs, 52 differentially expressed miRNAs, and 34 differentially expressed tRFs and tiRNAs between the two groups (Figures $1 \mathrm{~A}, \mathrm{~B}$ ). We found nine types of tRFs and tiRNAs in both groups, including tRF-1, tRF-2, tRF-3a, tRF-3b, tRF-5a, tRF-5b, tRF-5c, tiRNA-3, and tiRNA-5 (Figures 2 A-C). As shown in Figures $1 \mathrm{~A}$ and $\mathrm{B}$, compared with controls, patients had 33 upregulated miRNAs, 24 upregulated tRFs and tiRNAs, 19 downregulated miRNAs, and 10 downregulated tRFs and tiRNAs (fold change, cutoff 1.5; $p$-value, cutoff 0.05).

\section{qRT-PCR validation}

Next, in order to validate differential expression, we used qPCR to measure three strong candidates, tRF-Leu-TAA-006, tRF-GIn-TTG-033, and tRF-Lys-TTT-028, in the 20 patient samples and 20 control samples. The results showed that the expression trend of these three tRFs was consistent with the sequencing results; the difference was statistically significant (Figure 3).

Predictive value of specific tRFs and tiRNAs as diagnostic biomarkers of pediatric intussusception

The ROC curves for differentiating between pediatric-intussusception patients and healthy controls were based on expression levels of tRF-LeuTAA-006, tRF-GIn-TTG-033 and tRF-Lys-TTT-028. tRFs and tiRNAs with AUC $>0.5$ and $p<0.05$ were considered appropriate biomarkers. The results showed that the AUCs of tRF-Leu-TAA-006, tRF-Gln-TTG-033 and tRF-Lys-TTT-028 were 0.984 (95\% confidence interval [Cl]: 0.952-1.000; $p<0.05), 0.970$ (95\% Cl: 0.933-1.000; $p<0.05)$ and 0.837 (95\% Cl: $0.722-0.951 ; p<0.05)$, respectively (Figure 4).

\section{Prediction of potential targets for differentially expressed tRFs and tiRNAs and functional analysis of target genes}

We performed GO and KEGG pathway analyses to analyze target genes in order to explore the potential functions and mechanisms of tRFs and tiRNAs in pediatric intussusception. Classification of tRF and tiRNA target genes was based on the cellular component (CC), molecular function (MF), and biological process (BP) categories. Gene Ontology annotations demonstrated that the target genes of tRF-GIn-TTG-033 were abundant in the function of material metabolism, such as the nucleic acid metabolic process, heterocycle metabolic process, organonitrogen compound metabolic process, nitrogen compound metabolic process, and organic-substance metabolic process (Figure $5 \mathrm{~A}$ ). In addition, the target genes of tRF-GIn-TTG-033 were primarily found in the organelle, intracellular compartment, and cell part, while the MFs of its 
Table I. Demographic and clinical data of pediatric intussusception

\begin{tabular}{|c|c|c|c|c|c|c|}
\hline $\begin{array}{l}\text { Patient } \\
\text { No. }\end{array}$ & $\begin{array}{c}\text { Age } \\
\text { [months] }\end{array}$ & Gender & Pathological type & $\begin{array}{c}\text { Successful vs. } \\
\text { failed air enemas } \\
\text { for intussuscep- } \\
\text { tion }\end{array}$ & Mass location & $\begin{array}{l}\text { Signs of plain } \\
\text { radiography }\end{array}$ \\
\hline 1 & 11 & male & ileum colon & success & $\begin{array}{l}\text { hepatic flexure } \\
\text { of colon }\end{array}$ & $\begin{array}{l}\text { quadrant gas-filled } \\
\text { bowel loop }\end{array}$ \\
\hline 2 & 19 & female & ileum colon & success & cecum & paucity of bowel gas \\
\hline 3 & 15 & male & ileum colon & success & $\begin{array}{l}\text { transverse } \\
\text { colon }\end{array}$ & paucity of bowel gas \\
\hline 4 & 9 & female & $\begin{array}{l}\text { ileum-ileum, } \\
\text { duplication of } \\
\text { intestine }\end{array}$ & failed, operative & $\begin{array}{l}\text { hepatic flexure } \\
\text { of colon }\end{array}$ & $\begin{array}{c}\text { Distended bowel with } \\
\text { a mass }\end{array}$ \\
\hline 5 & 60 & male & ileum colon & success & $\begin{array}{l}\text { hepatic flexure } \\
\text { of colon }\end{array}$ & $\begin{array}{l}\text { quadrant gas-filled } \\
\text { bowel loop }\end{array}$ \\
\hline 6 & 6 & male & ileum colon & success & $\begin{array}{l}\text { hepatic flexure } \\
\text { of colon }\end{array}$ & $\begin{array}{c}\text { distended bowel with } \\
\text { a mass }\end{array}$ \\
\hline 7 & 24 & female & ileum colon & success & $\begin{array}{l}\text { hepatic flexure } \\
\text { of colon }\end{array}$ & $\begin{array}{l}\text { quadrant gas-filled } \\
\text { bowel loop }\end{array}$ \\
\hline 8 & 10 & male & ileum cecum & success & cecum & $\begin{array}{c}\text { distended bowel with } \\
\text { a mass }\end{array}$ \\
\hline 9 & 24 & male & ileum colon & success & $\begin{array}{l}\text { hepatic flexure } \\
\text { of colon }\end{array}$ & paucity of bowel gas \\
\hline 10 & 24 & female & ileum colon & success & $\begin{array}{l}\text { hepatic flexure } \\
\text { of colon }\end{array}$ & $\begin{array}{l}\text { quadrant gas-filled } \\
\text { bowel loop }\end{array}$ \\
\hline 11 & 36 & female & ileum colon & success & $\begin{array}{l}\text { hepatic flexure } \\
\text { of colon }\end{array}$ & $\begin{array}{l}\text { quadrant gas-filled } \\
\text { bowel loop }\end{array}$ \\
\hline 12 & 19 & male & ileum cecum & success & cecum & $\begin{array}{l}\text { quadrant gas-filled } \\
\text { bowel loop }\end{array}$ \\
\hline 13 & 36 & female & ileum colon & success & $\begin{array}{l}\text { hepatic flexure } \\
\text { of colon }\end{array}$ & paucity of bowel gas \\
\hline 14 & 72 & male & ileum-ileum & failed, operative & $\begin{array}{l}\text { hepatic flexure } \\
\text { of colon }\end{array}$ & $\begin{array}{l}\text { quadrant gas-filled } \\
\text { bowel loop }\end{array}$ \\
\hline 15 & 120 & male & ileum colon & success & $\begin{array}{l}\text { hepatic flexure } \\
\text { of colon }\end{array}$ & $\begin{array}{l}\text { quadrant gas-filled } \\
\text { bowel loop }\end{array}$ \\
\hline 16 & 1 & female & ileum colon & success & $\begin{array}{l}\text { hepatic flexure } \\
\text { of colon }\end{array}$ & $\begin{array}{l}\text { quadrant gas-filled } \\
\text { bowel loop }\end{array}$ \\
\hline 17 & 156 & male & ileum colon & success & $\begin{array}{l}\text { hepatic flexure } \\
\text { of colon }\end{array}$ & paucity of bowel gas \\
\hline 18 & 15 & female & ileum colon & success & $\begin{array}{l}\text { hepatic flexure } \\
\text { of colon }\end{array}$ & $\begin{array}{l}\text { quadrant gas-filled } \\
\text { bowel loop }\end{array}$ \\
\hline 19 & 12 & female & ileum colon & success & $\begin{array}{l}\text { hepatic flexure } \\
\text { of colon }\end{array}$ & $\begin{array}{l}\text { quadrant gas-filled } \\
\text { bowel loop }\end{array}$ \\
\hline 20 & 31 & female & ileum cecum & success & cecum & paucity of bowel gas \\
\hline
\end{tabular}

products mainly included binding, protein binding, and transferase activity (Figure 5 B). After mapping the targeted genes of tRF-GIn-TTG-033 in the KEGG database, we found that these genes participated in the "AMPK signaling pathway", "FoxO signaling pathway", "Cellular senescence" and "Relaxin signaling pathway", which were associated with I/R (Figure $5 \mathrm{C}$ ).

Regarding tRF-Leu-TAA-006, BP-based terms revealed that tRF target genes were related to the cellular protein modification process and the protein modification process (Figure 6 A). In addition, the target genes of tRF-Leu-TAA-006 were primarily found in the intracellular membrane-bound organelle, intracellular compartment, and cell part; and the MFs of its products mainly included binding, protein binding and ion binding (Figure 6 B). After mapping the targeted genes of tRF-LeuTAA-006 in the KEGG database, we found that these genes participated in the "RAS pathway" and "Protein procession", which were associated with I/R (Figure 6 C).

For tRF-Lys-TTT-028, target genes played a role in lipid metabolic processes, including the nucle- 

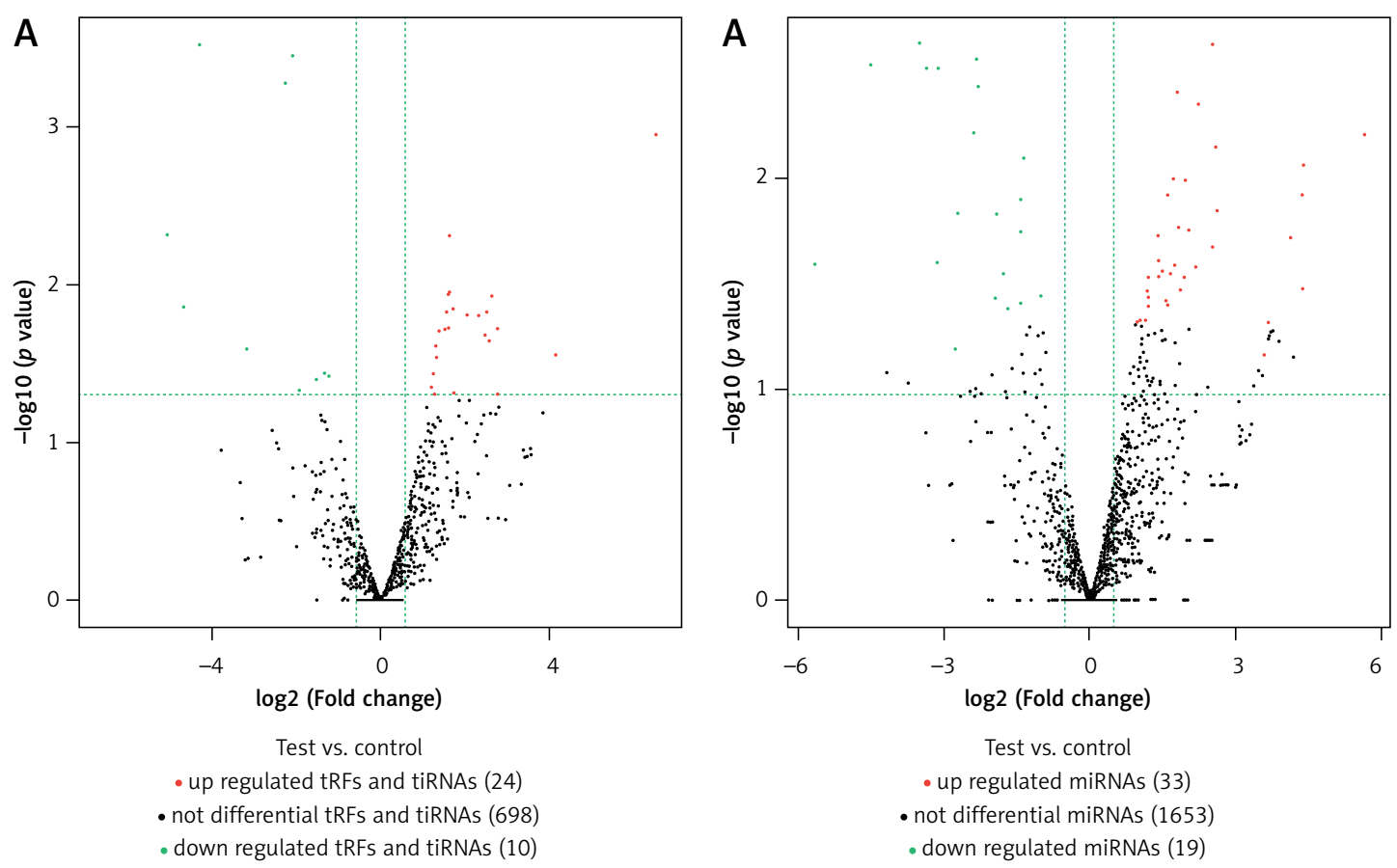

Figure 1. Differentially expressed tRFs (A) and miRNAs (B) between pediatric intussusception patients and healthy controls

A

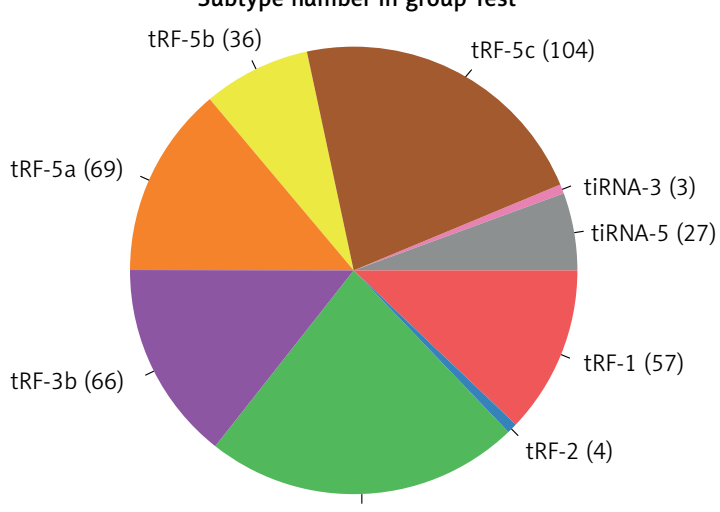

tRF-3a (108)

चtRF-1 $\square$ tRF-2
B

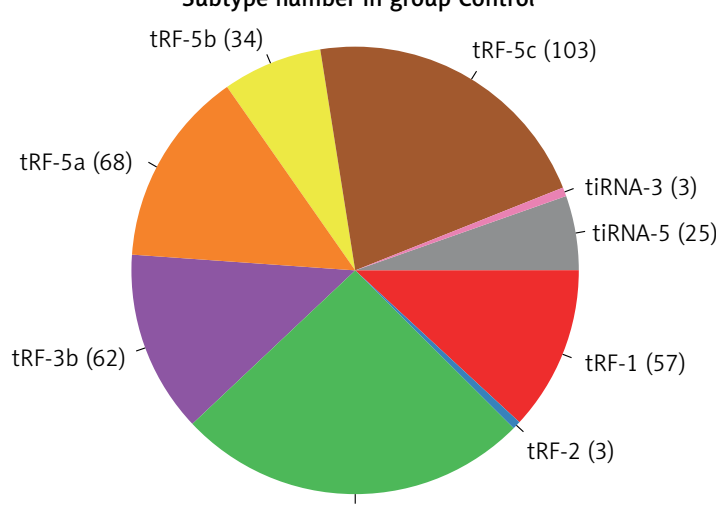

tRF-3a (121)

tRF-5b $\quad$ tRF-5c $\square$ tiRNA-3 $\square$ tiRNA-5

C

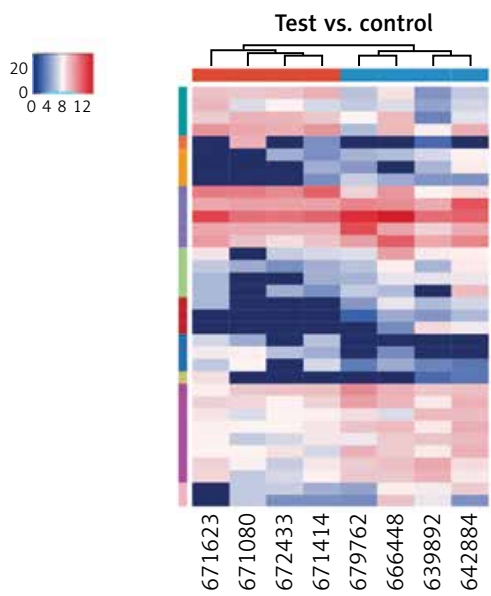

Figure 2. Subtypes of tRFs (A), tiRNAs (B) and heatmap (C) in patients and controls 

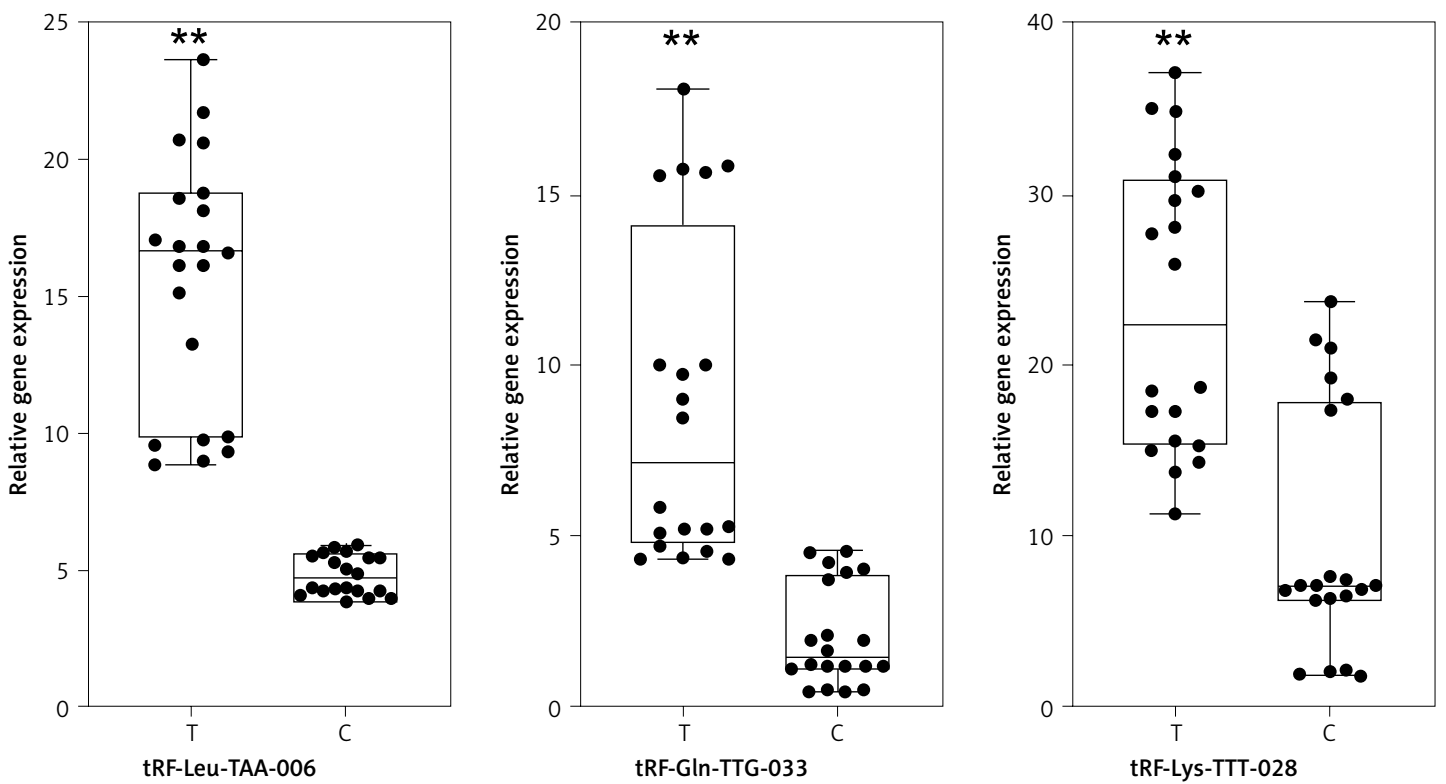

Figure 3. Verification of differentially expressed tRFs between patients and controls using qRT-PCR ${ }^{* *} p<0.01$.

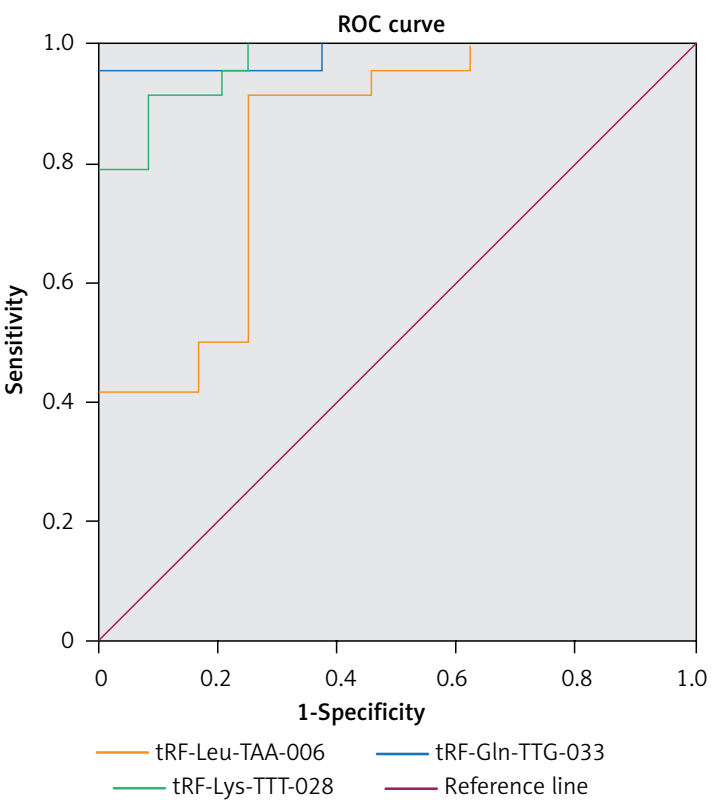

Figure 4. ROC curves of differentially expressed tRF-Leu-TAA-006, tRF-GIn-TTG-033 and tRF-LysTTT-028 between patients and controls. The AUCS of tRF-Leu-TAA-006, tRF-GIn-TTG-033 and tRF-LysTTT-028 were $0.984,0.970$ and 0.837 , respectively

ic acid metabolic process, macromolecule metabolic process, cellular macromolecule metabolic process, and RNA metabolic process (Figure $7 \mathrm{~A}$ ). In addition, these genes were primarily found in the intracellular membrane-bound organelle, intracellular compartment, and cell part, and the MFs of its products mainly included DNA binding, heterocyclic compound binding, and organic cyclic-compound binding (Figure 7 B). After mapping the targeted genes of tRF-Lys-TTT-028 in the KEGG database, we found that these genes participated in "Signaling pathways regulating pluripotency of stem cells" and "Taurine and hypotaurine metabolism”, which were associated with I/R (Figure 7 C).

\section{tRF-target gene interaction network analysis}

Having revealed the correlation between tRFs and pediatric intussusception, we next explored interactions between target genes predicted by our biological analysis to better understand the intrinsic mechanisms of tRFs. Using the Miranda (http:// www.microrna.org/) and RNAhybrid (M. Rehmsmeier, 2004) tools, we found the top 100 potential targets of tRF-Leu-TAA-006, tRF-Gln-TTG-033, and TRF-Lys-TTT-028, as shown respectively in Figures $8 \mathrm{~A}-\mathrm{C}$.

\section{Discussion}

Intussusception is one of the most common causes of bowel obstruction in children. I/R in intussusception can result in mucosal erosion and hemorrhagic ulceration, as well as necrosis of the bowel, lung injury, and multiple-organ dysfunction syndrome [21]. Oxidative stress can play a crucial role in I/R in pediatric intussusception [22]. Some research shows that early intervention has a protective effect in I/R $[23,24]$. So early detection of intestinal I/R in intussusception can improve prognosis and reduce complications in the intestines and extraintestinal organs. Although many efforts have been made to identify biomarkers of pediatric intussusception and associated I/R, no defi- 

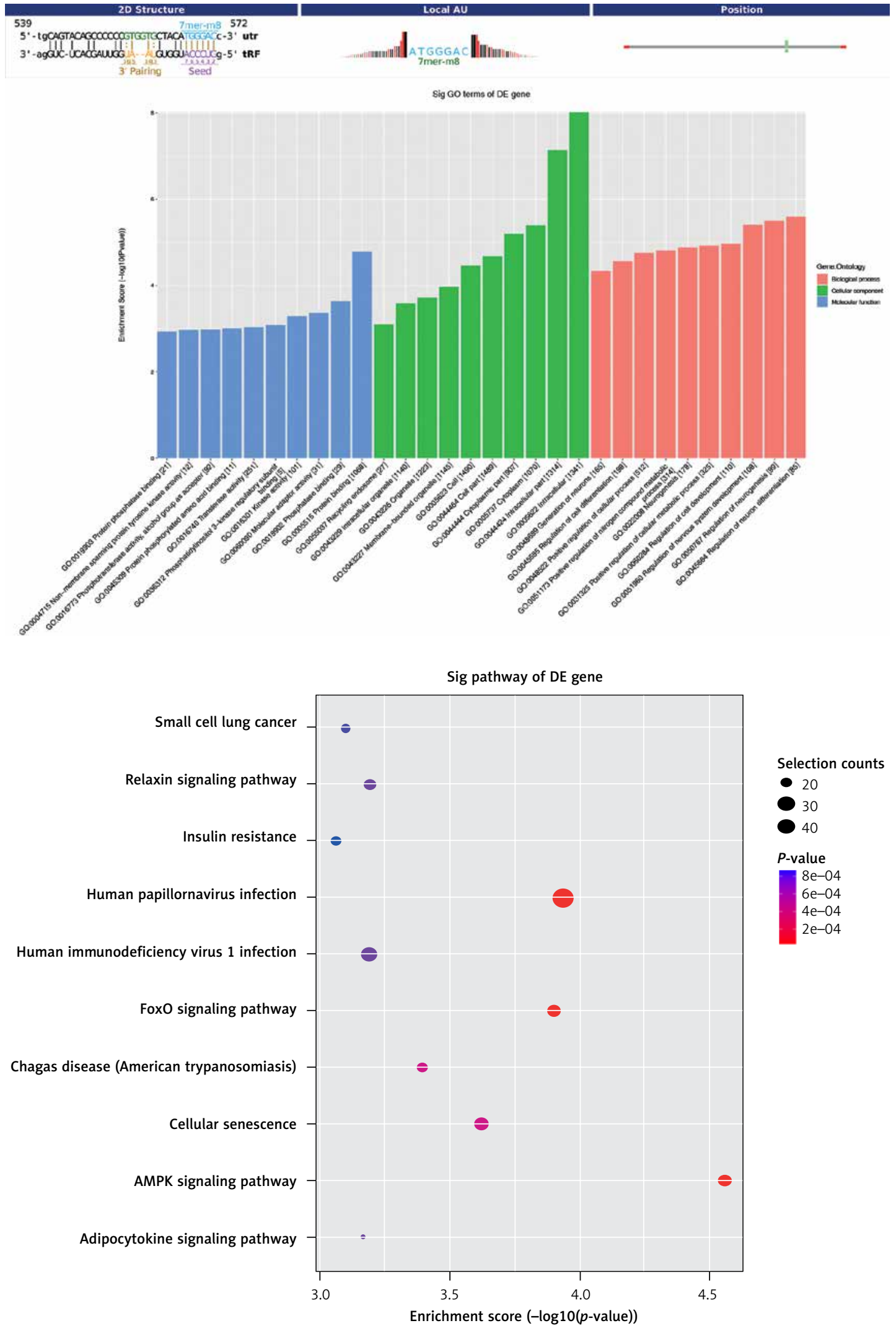

Figure 5. Gene Ontology (GO) and pathway analysis of tRF-GIn-TTG-033. Target genes were predicted by Miranda algorithms and TargetScan miRNA prediction programs 

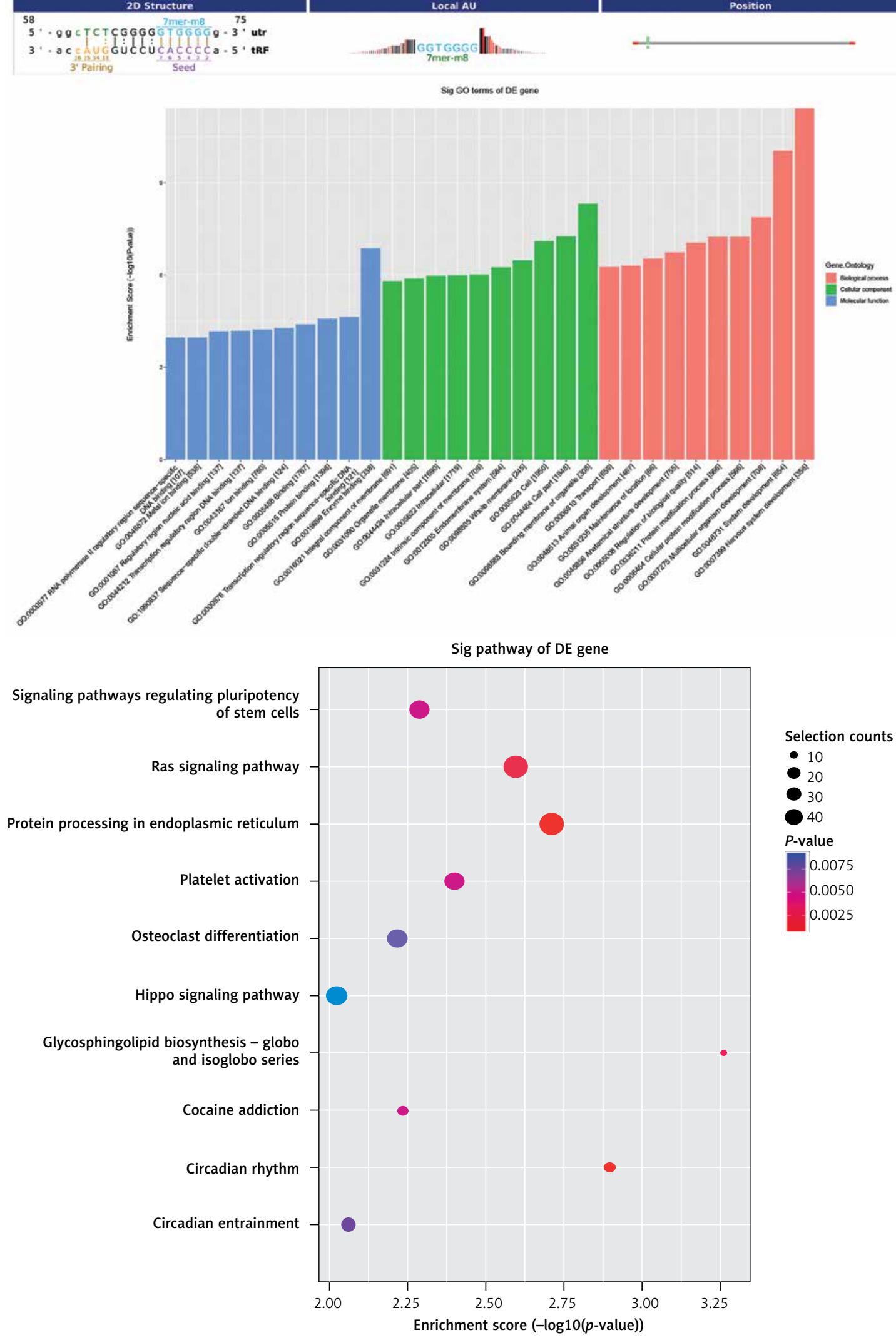

Figure 6. Gene Ontology (GO) and pathway analysis of tRF-Leu-TAA-006. Target genes were predicted by Miranda algorithms and TargetScan miRNA prediction programs 

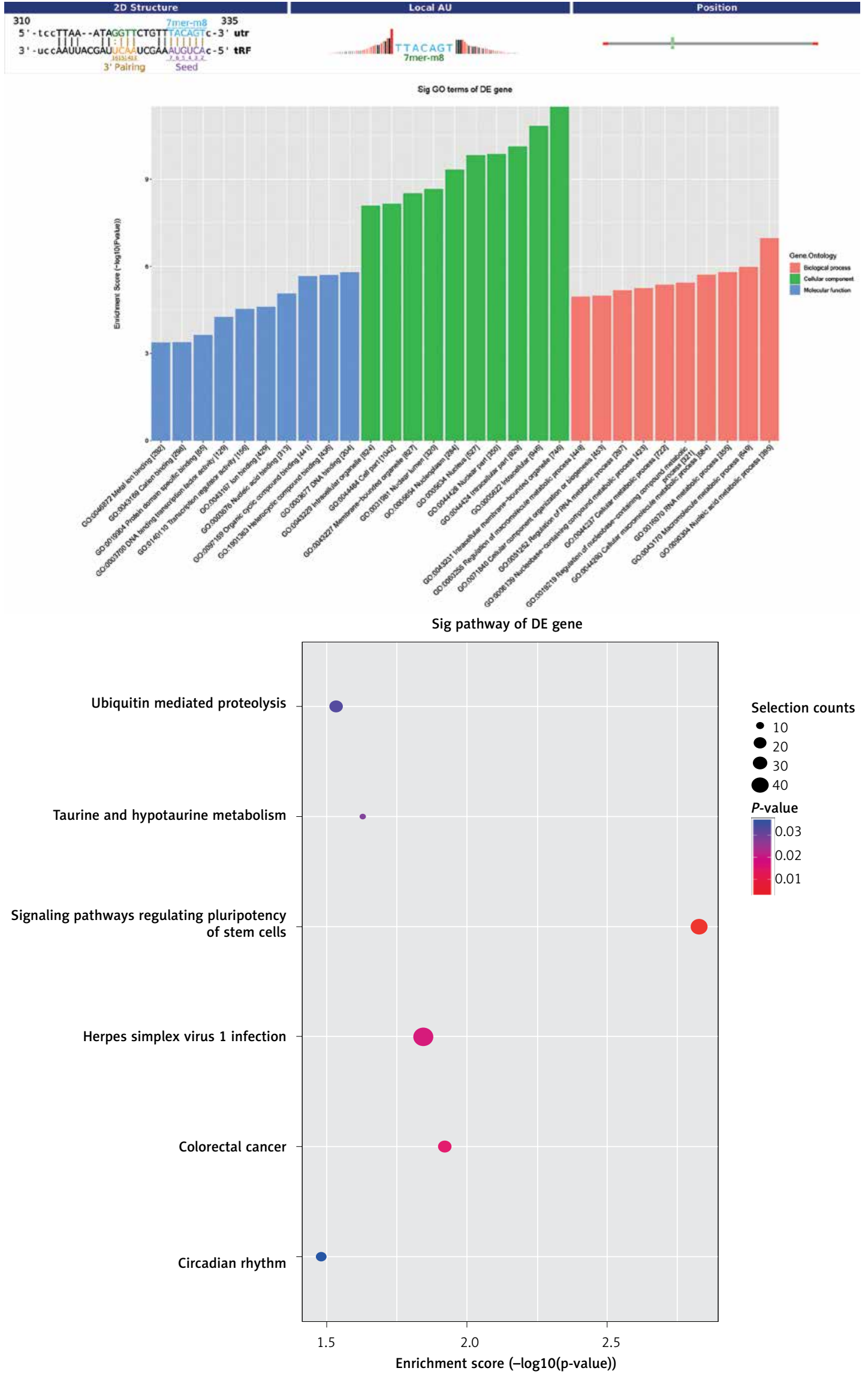

Figure 7. Gene Ontology (GO) and pathway analysis of tRF-Lys-TTT-028. Target genes were predicted by Miranda algorithms and TargetScan miRNA prediction programs 
A

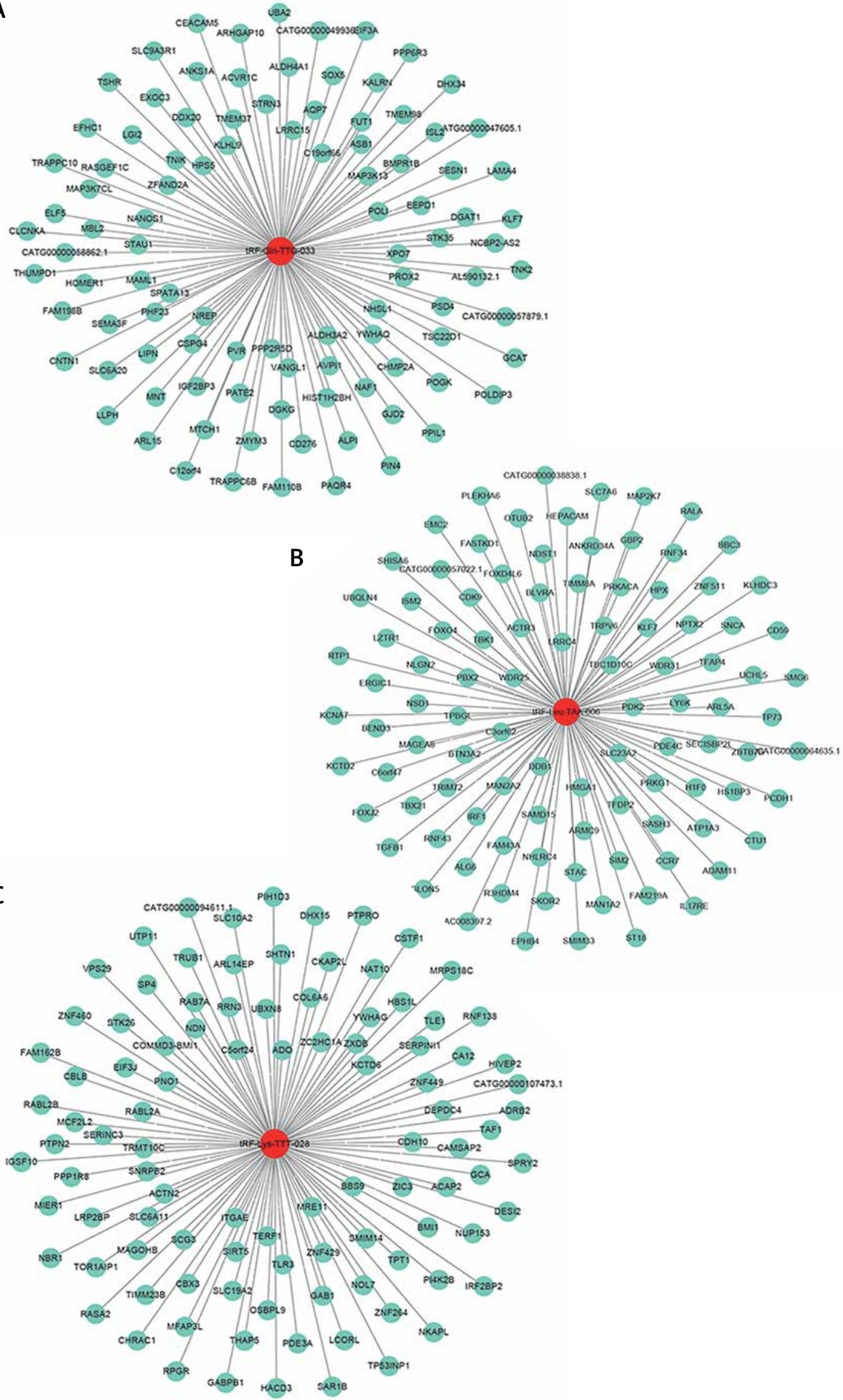

Figure 8. Top 100 potential targets of tRF-Leu-TAA-006 (A), tRF-Gln-TTG-033 (B) and tRF-Lys-TTT-028 (C) 
nite biomarkers are currently available in clinical practice.

Non-coding RNAs (ncRNAs) can be released to circulate in the peripheral blood. Some studies have shown that tRFs are related to translation, gene expression, and the cellular stress response. The mechanisms underlying tRFs regulate some human diseases, such as viral infections, cancers, pathological stress damage, neurological diseases and metabolic diseases $[12,15]$. Circulating levels of tRFs could be useful as biomarkers of some diseases and surrogate measures of disease progression $[13,14]$, indicating their potential use in diagnosis and assessment of $\mathrm{I} / \mathrm{R}$ in pediatric intussusception. In our previous studies, we found I/R in both our animal models and our pediatric intussusception patients. The diagnosis of existing intussusception cannot provide the clues for precise reflection of how tRFs are related to translation, gene expression, and the cellular stress response. Therefore, we decided to test the expression of tRFs in serum from patients and further focus on biological analysis to verify whether circulating tRFs could serve as potential biomarkers in pediatric intussusception and associated I/R.

In the present study, we first performed high-throughput sequencing (HTS) to detect the expression profiles of tRFs in pediatric intussusception patients. To validate differential expression in the sequencing data, we selected three dysregulated tRFs for QRT-PCR review to verify the authenticity of the profiles. We detected the most significantly upregulated tRFs-tRF-LeuTAA-006, tRF-Gln-TTG-033, and tRF-Lys-TTT-028 - in serum samples from patients. Our qRT-PCR results demonstrated that all three tRFs were significantly upregulated in patients compared with controls. Via GO analysis, we learned that the targeted genes of these differentially expressed tRFs were involved in metabolism, protein modification, binding, transferase activity, protein binding, DNA binding, heterocyclic compound binding, and organic cyclic-compound binding. In mapping all targeted genes to the KEGG database, we noted that they participated in the following: "AMPK signaling pathway", "FoxO signaling pathway", "Cellular senescence", "Relaxin signaling pathway", "RAS pathway", "Protein procession", "Signaling pathways regulating pluripotency of stem cells" and "Taurine and hypotaurine metabolism". This is consistent with findings in the literature that these signaling pathways play important roles in I/R [25-32]. However, the involvement of these dysregulated tRFs and their target genes in I/R in the pediatric intussusception process via these signaling pathways requires further studies.

To apply the expression values of serum tRFs and tiRNAs for clinical diagnosis, we analyzed the ROC curves. We found that serum tRF-Leu-
TAA-006, tRF-GIn-TTG-033, and tRF-Lys-TTT-028 were significantly upregulated in pediatric intussusception children compared with controls. The results showed that AUCs of tRF-Leu-TAA-006, tRF-GIn-TTG-033, and tRF-Lys-TTT-028 were 0.984, 0.970 , and 0.837, respectively (Figure 5). These findings suggested that serum levels of these three tRFs might be involved in the development of pediatric intussusception and could become new biomarkers for pediatric intussusception. The present study found higher levels of tRFs, the targeted genes of these differentially expressed tRFs and the associated pathway. Thus, it may provide clues for precise decision making for treatment of intussusception associated with I/R.

There are some limitations of the present study. First, the sample size was relatively small. Second, only three tRFs identified in microarray experiments were validated via qRT-PCR. Future studies should be conducted with larger sample sizes and in-depth verification of more candidate genes. Finally, the potential mechanisms of tRF-LeuTAA-006, tRF-GIn-TTG-033, and tRF-Lys-TTT-028 in pediatric intussusception and how the changes of tRFs targeting pathways affect the progression of the disease still require further studies.

In conclusion, the present study provided an overall analysis of tRFs and tiRNAs in pediatric intussusception and indicated that tRF-LeuTAA-006, tRF-GIn-TTG-033, and tRF-Lys-TTT-028 could play important roles in pediatric intussusception. The results of further biological analysis suggested that these three tRFs could serve as novel serological biomarkers with significant accuracy in diagnosing pediatric intussusception. We believe that our research could offer clues for further research into the mechanism of tRFs in pediatric intussusception.

\section{Conflict of interest}

The authors declare no conflict of interest.

\section{References}

1. Jiang J, Jiang B, Parashar U, Nguyen T, Bines J, Patel MM. Childhood intussusception: a literature review. PLOS One 2013; 8: e68482.

2. Guo WL, Hu ZC, Tan YL, Sheng M, Wang J. Risk factors for recurrent intussusception in children: a retrospective cohort study. BMJ Open 2017; 7: e018604.

3. Guo WL, Wang J, Liu C, Yang FB, Li SW. Expression of p38 mitogen-activated protein kinase (p38MAPK) and pathological change in intussusception. Pediatr Int 2016; 58: 881-6.

4. Hu ZC, Tan YL, Huang SG, Peng Pan 1, Liu XB, Wang J, et al. Molecular imaging of Toll-like receptor 4 detects ischemia-reperfusion injury during intussusception. Oncotarget 2018; 9: 7882-90.

5. Hanquinet S, Anooshiravani M, Vunda A, Le Coultre C, Bugmann P. Reliability of color Doppler and power Dop- 
pler sonography in the evaluation of intussuscepted bowel viability. Pediatr Surg Int 1998; 13: 360-2.

6. Guo WL, Wang J, Zhou M, Sheng M, Fang L. The role of plain radiography in assessing intussusception with vascular compromise in children. Arch Med Sci 2011; 7: 877-81.

7. Carroll AG, Kavanagh RG, Leidhin CN, Cullinan NM, Lavelle LP, Malone DE. Comparative effectiveness of imaging modalities for the diagnosis and treatment of intussusception: a critically appraised topic. Acad Radiol 2017; 24: 521-9.

8. Dörterler ME, Kocaman OH. Selection of pneumatic reduction in invagination treatment and the factors affecting the success of this method. Cureus 2019; 11: e5928.

9. McDermott VG, Taylor T, Mackenzie S, Hendry GM Pneumatic reduction of intussusception: clinical experience and factors affecting outcome. Clin Radiol 1994; 49: 30-4.

10. Soares AR, Santos M. Discovery and function of transfer RNA-derived fragments and their role in disease. Wiley Interdiscip Rev RNA 2017; 8.

11. Shen L, Gan M, Tan Z, Jiang D, Jiang Y, Li M, et al. A novel class of tRNA-derived small non-coding RNAs respond to myocardial hypertrophy and contribute to intergenerational inheritance. Biomolecules 2018; 8: 54.

12. Shen Y, Yu X, Zhu L, Li T, Yan Z, Guo J. Transfer RNA-derived fragments and tRNA halves: biogenesis, biological functions and their roles in diseases. J Mol Med (Berl) 2018; 96: 1167-76.

13. Huang Y, Ge H, Zheng M, Cui Y, Fu Z, Wu X, et al. Serum tRNA-derived fragments (tRFs) as potential candidates for diagnosis of nontriple negative breast cancer. J Cell Physiol 2020; 235: 2809-24.

14. Tosar JP, Cayota A. Extracellular tRNAs and tRNA-derived fragments. RNA Biol 2020; 19: 1149-67.

15. Mishima E, Inoue C, Saigusa D, Inoue R, Ito K, Suzuki Y, et al. Conformational change in transfer RNA is an early indicator of acute cellular damage. J Am Soc Nephrol 2014; 25: 2316-26.

16. Andrews S. FastQC: A Quality Control tool for High Throughput Sequence Data. Babraham Bioinformatics 2010, http://www.bioinformatics.babraham.ac.uk/projects/fastqc.

17. Martin M. Cutadapt removes adapter sequences from high-throughput sequencing reads. EMBnet.journal 2011; 17: 10-2.

18. Langmead B, Trapnell C, Pop M, Salzberg SL. Ultrafast and memory-efficient alignment of short DNA sequences to the human genome. Genome Biol 2009; 10: R25.

19. Friedländer MR, Mackowiak SD, Li N, Chen W, Rajewsky N. miRDeep2 accurately identifies known and hundreds of novel microRNA genes in seven animal clades. Nucleic Acids Res 2012; 40: 37-52.

20. Robinson MD, McCarthy DJ, Smyth GK. edgeR: a Bioconductor package for differential expression analysis of digital gene expression data. Bioinformatics 2010; 26: 139-40.

21. Pierro A, Eaton S. Intestinal ischemia reperfusion injury and multisystem organ failure. Semin Pediatr Surg 2004; 13: 11-7.

22. Wu MJ, Chen M, Sang S, Hou LL, Tian ML, Li K, et al. Protective effects of hydrogen rich water on the intestinal ischemia/reperfusion injury due to intestinal intussusception in a rat model. Med Gas Res 2017; 7: 101-6.

23. LU Y, Xi J, Zhang Y, Li C, Chen W, Hu X, et al. MicroRNA-214-5p protects against myocardial ischemia reper- fusion injury through targeting the FAS ligand. Arch Med Sci 2020; 16: 1119-29.

24. Sayhan MB, Oguz S, Salt Ö, Can N, Ozgurtas T, Yalta TD. Sesamin ameliorates mucosal tissue injury of mesenteric ischemia and reperfusion in an experimental rat model. Arch Med Sci 2019; 15: 1582-18.

25. Liu Z, Chen JM, Huang H, Kuznicki M, Zheng S, Sun W, et al. The protective effect of trimetazidine on myocardial ischemia/reperfusion injury through activating AMPK and ERK signaling pathway. Metabolism 2016; 65: $122-30$

26. Clements ME, Chaber CJ, Ledbetter SR, Zuk A. Increased cellular senescence and vascular rarefaction exacerbate the progression of kidney fibrosis in aged mice following transient ischemic injury. PLoS One 2013; 8: e70464.

27. Juhasz B, Thirunavukkarasu M, Pant R, Zhan L, Penumathsa SV, Secor ER, et al. Bromelain induces cardioprotection against ischemia-reperfusion injury through Akt/FOXO pathway in rat myocardium. Am J Physio Heart Circ Physiol 2008; 294: H1365-70.

28. Pando R, Cheporko Y, Haklai R, Maysel-Auslender S, Keren G, George J, et al. Ras inhibition attenuates myocardial ischemia-reperfusion injury. Biochem Pharmacol 2009; 77: 1593-601

29. Liu H, Wang L, Weng X, Chen H, Du Y, Diao C, et al. Inhibition of Brd4 alleviates renal ischemia/reperfusion injury-induced apoptosis and endoplasmic reticulum stress by blocking FoxO4-mediated oxidative stress. Redox Biol 2019; 24: 101195.

30. Jouret F, Leenders J, Poma L, Defraigne JO, Krzesinski JM, de Tullio P. Nuclear magnetic resonance metabolomic profiling of mouse kidney, urine and serum following renal ischemia/reperfusion injury. PLoS One 2016; 11: e0163021.

31. Zhang P, Ming Y, Cheng K, Niu Y, Ye Q. Gene expression profiling in ischemic postconditioning to alleviate mouse liver ischemia/reperfusion injury. Int J Med Sci 2019; 16: 343-54.

32. Valle Raleigh J, Mauro AG, Devarakonda T, Marchetti C, He J, Kim E, et al. Reperfusion therapy with recombinant human relaxin-2 (Serelaxin) attenuates myocardial infarct size and NLRP3 inflammasome following ischemia/reperfusion injury via eNOS-dependent mechanism. Cardiovasc Res 2017; 113: 609-19. 